NIST Special Publication 1200-2

\title{
Preparation of Nanoparticle Dispersions from Powdered Material Using Ultrasonic Disruption \\ Version 1.1
}

J. S. Taurozzi

V. A. Hackley

National Institute of Standards and Technology

Material Measurement Laboratory Gaithersburg, MD 20899-8520

M. R. Wiesner

Duke University

Department of Civil and Environmental Engineering

Durham, NC 27708

http://dx.doi.org/10.6028/NIST.SP.1200-2
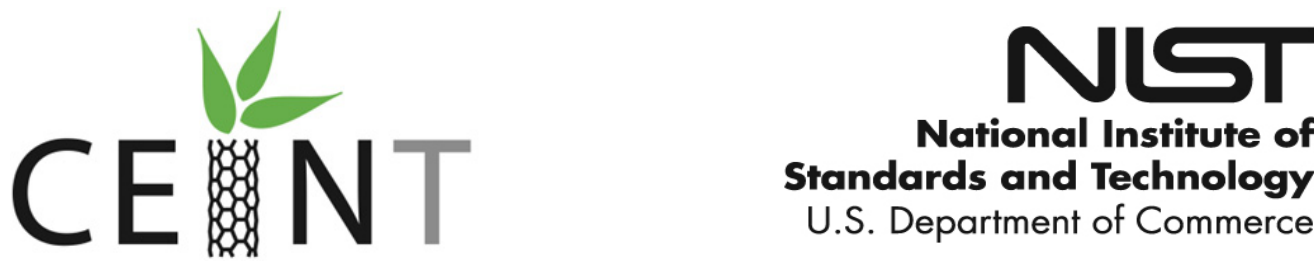

National Institute of Standards and Technology U.S. Department of Commerce 


\section{Preparation of Nanoparticle Dispersions from Powdered Material Using Ultrasonic Disruption}

Version 1.1

J. S. Taurozzi

V. A. Hackley

National Institute of Standards and Technology Material Measurement Laboratory Gaithersburg, MD 20899-8520

M. R. Wiesner Duke University Department of Civil and Environmental Engineering

Durham, NC 27708

http://dx.doi.org/10.6028/NIST.SP.1200-2

June 2012

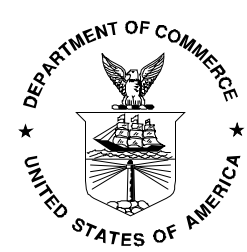

U.S. Department of Commerce John E. Bryson, Secretary 
Patrick D. Gallagher, Under Secretary of Commerce for Standards and Technology and Director 
Certain commercial entities, equipment or materials may be identified in this document in order to describe an experimental procedure or concept adequately. Such identification is not intended to imply recommendation or endorsement by the National Institute of Standards and Technology, nor is it intended to imply that the entities, materials or equipment are necessarily the best available for the purpose.

National Institute of Standards and Technology Special Publication 1200-2 Natl. Inst. Stand. Technol. Spec. Publ. 1200-2, 14 pages (June 2012) CODEN: NSPUE 


\section{Foreword}

This special publication is one in a series of protocols resulting from a collaborative research agreement between the National Institute of Standards and Technology (NIST) and Duke University's Center for the Environmental Implications of Nanotechnology (CEINT). The original version of this protocol (Ver. 1.0) was first posted on the CEINT web site (http://ceint.duke.edu) and it, along with any other previous version, is superseded by this updated special publication version. Updates to this protocol may be released in the future. Visit http://nist.gov/mml/np-measurement-protocols.cfm to check for revisions of this protocol or new protocols in the series.

NIST and CEINT are interested in soliciting feedback on this method. We value user comments and suggestions to improve or further validate this protocol. Please send your name, email address and comments/suggestions to nanoprotocols@nist.gov. We also encourage users to report citations to published work in which this protocol has been applied. 


\section{Introduction}

Toxicity and fate assessment are key elements in the evaluation of the environmental, health and safety risks of engineered nanomaterials (ENMs). While significant effort and resources have been devoted to the toxicological evaluation of many ENMs, obtaining conclusive and reproducible results continues to be a challenge. This can be traced in part to the lack of standardized dispersion protocols and the inconsistent application of dispersion procedures in relevant biological and environmental matrices $(1,2)$. In order to address these issues, the National Institute of Standards and Technology (NIST) jointly with the Center for the Environmental Implications of Nanotechnology (CEINT) have developed a series of standardized and validated protocols for the dispersion of ENMs from a powdered material source for both human health and environmental testing applications.

\section{Principles and scope}

This protocol is generic in nature and is intended to provide relevant guidelines for the preparation of nanoparticle dispersions in liquid media by the application of ultrasonic energy (a process referred to here as sonication). It applies specifically to engineered nanoscale materials (ENM) obtained in dry powder form, but can be extended to the treatment of previously suspended ENMs (e.g., for purposes of re-dispersion).

For a more in-depth discussion of specific parameters addressed in this protocol, the reader is advised to consult reference (2). For guidelines on reporting relevant conditions and critical parameters relating to the ultrasonic dispersion of ENMs, refer to (3). For additional relevant and general considerations on the application of ultrasound, the reader is urged to consult the references (4-11).

\section{General considerations}

Ultrasonic waves are generated in a liquid suspension either by immersing an ultrasound probe or "horn" into the suspension (direct sonication), or by introducing the sample container with the suspension into a bath containing a liquid through which ultrasonic waves are propagated (indirect sonication).

In a sonication bath (indirect sonication), the ultrasonic waves must traverse the bath liquid and then pass through the wall of the sample container before reaching the suspension. In direct sonication, the probe is immersed directly into the suspension, reducing the physical barriers to delivering the power to the dispersion.

Direct sonication is recommended over indirect sonication for the purpose of dispersing dry powders, as it yields a higher effective energy output into the suspension. Indirect sonication can be used to re-suspend ENMs which have been pre-processed via direct sonication, or for ENMs that may be subject to unintended modifications or damage under direct sonication (e.g., cleavage of single wall carbon nanotubes).

Sonication is a highly system-specific dispersion procedure, involving a variety of concomitant complex physicochemical interactions that can result in either cluster breakdown or further agglomeration, as well as other effects including chemical reactions.

For a given system, optimal sonication conditions must be determined by assessing the effect of a variety of sonication parameters on the dispersion state of the suspension under a broad range 
of relevant conditions. Moreover, the total effective acoustic energy utilized in fragmenting powder clusters is influenced by a number of instrument- and system-specific parameters.

Adequate personal protective equipment (sound protection and respirator when applicable, goggles and lab coat) should be worn at all times. An acoustic enclosure is recommended for direct sonication procedures. A combination of ear plugs (recommended protection level NRR 30/SNR 34) and/or ear muffs should be used when in close proximity to the ultrasound source.

\section{Specific considerations}

\section{Temperature}

During sonication, the extreme local heating cycles that take place at the micro-scale bubble interface due to cavitation will result in a bulk heating of the liquid over time. Excessive bulk heating can cause substantial liquid evaporation, resulting in changes to the sonicated volume, or the degradation of the material or medium components.

A simple approach to minimize temperature driven side effects is to avoid substantial hightemperature excursions by immersing the suspension container in a cooling bath. The container should be immersed to a level roughly equal to that of the internal suspension.

An ice-water bath is often sufficient to prevent suspensions from overheating. If, however, ice water is not sufficient (e.g., the sample volume is too low, the sonication time is too long, or high sonication powers are used) an ice-salt bath can be used instead.

Working with containers made of materials with high thermal conductivities helps ensure a rapid release of heat from the suspension. The following container materials are suggested, in order of decreasing thermal conductivity: aluminum, stainless steel, glass, plastic. When selecting the container, consideration must also be given to the chemical compatibility between the container material and the suspension components. For instance, aluminum is incompatible with acidic suspensions and glass is incompatible with alkaline solutions.

Additionally, an aluminum foil or other cover with an opening just big enough to allow for the probe to pass through is recommended to reduce the evaporative loss of liquid content, especially when sonicating in volatile solvents (e.g., ethanol) or for substantial durations. Cooling the sample will also reduce evaporative loss. The volume of suspension before and after sonication should be verified and any corrections for evaporation reported. The cover will also minimize the potential release of aerosols generated by the sonication process.

\section{Sonication time and operation mode}

The total amount of energy (E) delivered to a suspension not only depends on the applied power (P) but also on the total amount of time (t) that the suspension is subject to the ultrasonic treatment: $E=P x t$. Consequently, two suspensions treated at the same power for different times can show significantly different dispersion states.

Ultrasonic disruptors can typically operate in either continuous or pulsed mode. In pulsed mode, ultrasonic intervals are alternated with static (sonication off) intervals. The duration of on and off intervals can be regulated. Operating in pulsed mode retards the rate of temperature increase in the medium, minimizing unwanted side effects and allowing for better temperature control than continuous mode operation. Pulse mode operation is therefore generally recommended. 


\section{Sample volume and concentration}

While sonication power and time describe the amount of energy delivered to the suspension, samples of different volumes and particle concentrations can respond differently to the same amount of delivered energy.

At constant volume, higher particle concentrations result in an increased particle collision frequency. In principle, an increased collision frequency can enhance particle breakage due to an increase in particle-particle impact events. However, if sufficient local activation and sintering energies are achieved, increased collision frequencies can also induce agglomerate or aggregate formation as particles collide and coalesce. The effect of concentration is thus dependent on both the energy delivered into the suspension and the physiochemical properties of the suspension.

At the particle concentrations commonly encountered in environmental and toxicological evaluations, no significant impact on the medium's acoustic properties is anticipated as a direct result of the particle phase.

The effect of suspension volume (at equal particle concentrations) is measured as energy density $(\mathrm{W} \cdot \mathrm{s} / \mathrm{mL})$. This magnitude expresses the amount of delivered energy per unit of suspension volume. In principle, at equal power and particle concentration, higher energy densities (i.e., lower suspension volumes) will result in a greater disruptive effect.

When working with small volumes the temperature of the suspension will rise more rapidly; therefore, more intense cooling conditions may be required.

\section{Sonicator probe, container geometry and tip immersion}

The sonicator probe (or horn) is the acoustic element that conducts the acoustic energy from the transducer into the suspension. The amount of acoustic energy transferred to the suspension will depend on the shape and diameter of the probe and its immersion depth in the suspension.

The vibrational amplitude of the probe at a given power setting is dependent on the probe taper. Usually, more pronounced tapers result in larger vibrational amplitudes and consequently a higher delivered power.

At equivalent instrumental power settings, microtip probes vibrate with larger amplitudes than conventional flat tip probes. However, microtips are less mechanically robust and are limited in terms of the maximum power setting at which they can be used. The manufacturer will typically specify a maximum power setting (based on a percentage of the maximum power output) for use with microtips. Microtips are appropriate for small suspension volumes (e.g., $25 \mathrm{~mL}$ or less).

The way in which the ultrasonic energy is distributed within the suspension is also heavily influenced by the container geometry. When possible, it is recommended to use the smallest diameter vessel that allows for the probe to be inserted without touching the container walls. Using smaller container diameters raises the height of the liquid and maximizes the liquidsurface area exposed to the acoustic waves, as well as the container wall surface/volume ratio for dissipation of heat by the cooling bath.

Conical bottom, flat bottom and round bottom flasks will show different energy maxima and minima distribution profiles, which will in turn vary significantly for different probe tapers and probe tip immersion depths. Cylindrical, flat bottom beakers are recommended, especially for small volumes. 
Probe immersion depths between ( 2 to 5 ) cm are recommended when operating with standard 1/2" probes having flat tips or with microtips. Probes should be placed no closer than about $1 \mathrm{~cm}$ from the bottom of the sample container and contact between the probe and the container walls should be avoided.

\section{Medium properties}

The ultrasonic energy delivered to a suspension is partly attenuated and dissipated by the suspending medium. Attenuation is determined by the medium's viscosity, density, acoustic wave speed and chemical composition, all of which impact on the amount of delivered energy that is effectively utilized to disrupt powder clusters. In principle, a higher medium viscosity will result in a greater acoustic attenuation. Additionally, higher viscosities will dampen the cavitation process, requiring higher power inputs to achieve dispersion. The medium's density and acoustic wave speed will also impact on the amount of vibrational energy that is transformed into acoustic energy.

For media relevant to biological testing, the salt content is likely to have a measurable impact on the acoustic properties relative to pure water, since the presence of physiological salt levels will slightly alter the density and viscosity of the solution. Overall, however, the effect of salt content is relatively small compared with other factors discussed previously.

\section{Calibration of delivered power}

\section{The calorimetric method}

Calorimetry is a relatively simple, fast and inexpensive procedure that allows for the direct measurement of the effective acoustic energy delivered to a sonicated liquid in a deviceindependent manner. The method is based on the measurement of the temperature increase in a liquid medium over time as a result of cavitation induced in a liquid by an immersed ultrasound probe.

At a given device output power setting, the temperature increase in the liquid is recorded over time and the effective delivered power can be calculated using the following equation:

$$
P=\frac{d T}{d t} M C_{p}
$$

where $P$ is the delivered acoustic power (W), $T$ and $t$ are temperature $(\mathrm{K})$ and time (s), respectively, $C_{p}$ is the specific heat of the liquid $(\mathrm{J} / \mathrm{g} \cdot \mathrm{K})$ and $M$ is the mass of liquid $(\mathrm{g})$.

The following steps are recommended for the determination of direct sonication calorimetric curves.

1. Fill a $600 \mathrm{~mL}$ cylindrical borosilicate beaker with $500 \mathrm{~mL}$ of de-ionized water (resistivity $\geq 18 \mathrm{M} \Omega . \mathrm{cm}$ )

2. Determine the mass of the liquid using a top loading balance (first tare the empty 600 $\mathrm{mL}$ beaker).

3. Immerse the sonicator probe (horn) approximately $2.5 \mathrm{~cm}(1 ")$ below the liquid surface 
4. Immerse a temperature probe connected to a temperature meter and data logger (e.g., an Extech HD $200^{\S}$ temperature meter and data logger coupled to a Type K immersion temperature probe). The probe tip should be about $1 \mathrm{~cm}$ away from the sonicator probe (Figure 1).

5. Select a sonicator output power setting and, operating in continuous mode, record the water temperature increase for the initial 5 minutes. During sonication, ensure that the beaker does not shift position, especially when operating at high power settings; this can be accomplished, for instance, by using a clamp attached to a ring stand.

6. Using the recorded values, create a temperature vs. time curve and obtain the best linear fit for the curve using least squares regression.

With the obtained slope, calculate the delivered power using Eq. 1. The procedure should be repeated for all power settings, relating each setting level to the measured power. The power obtained via calorimetry is the recommended reported power value for direct sonication-based dispersion procedures.

Using a different water volume, a container of a different material or a different type of probe than those recommended herein can result in changes in the measured temperature vs. time slope for a given power setting due to variations in the rate of heat transference between the sonicated water and the environment. If conditions other than those recommended herein are used to measure calorimetric curves, the altered conditions should be clearly reported.

Figure 2 shows calorimetric curves and calculated delivered powers at different device output settings, obtained using the above method for a Branson 450 analog disruptor using a standard $1 / 2$ " probe.

\footnotetext{
$\S$ The identification of any commercial product or trade name does not imply endorsement or recommendation by the National Institute of Standards and Technology.
} 


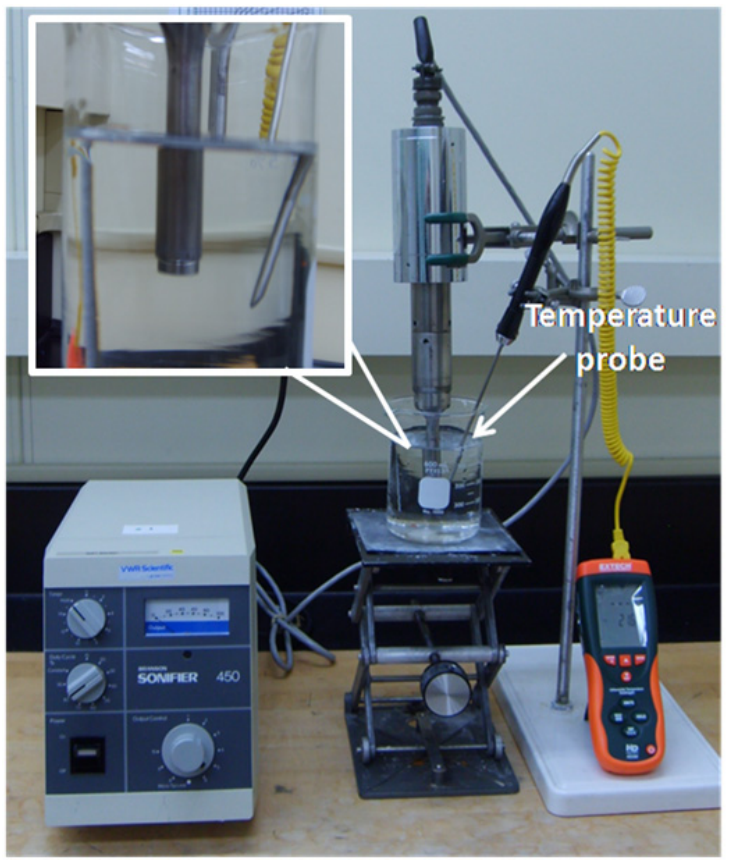

Figure 1. Setup for the measurement of calorimetric curves. Inset shows sonicator tip and temperature probe immersion depths. Sound protection enclosure was omitted for clarity of the image.

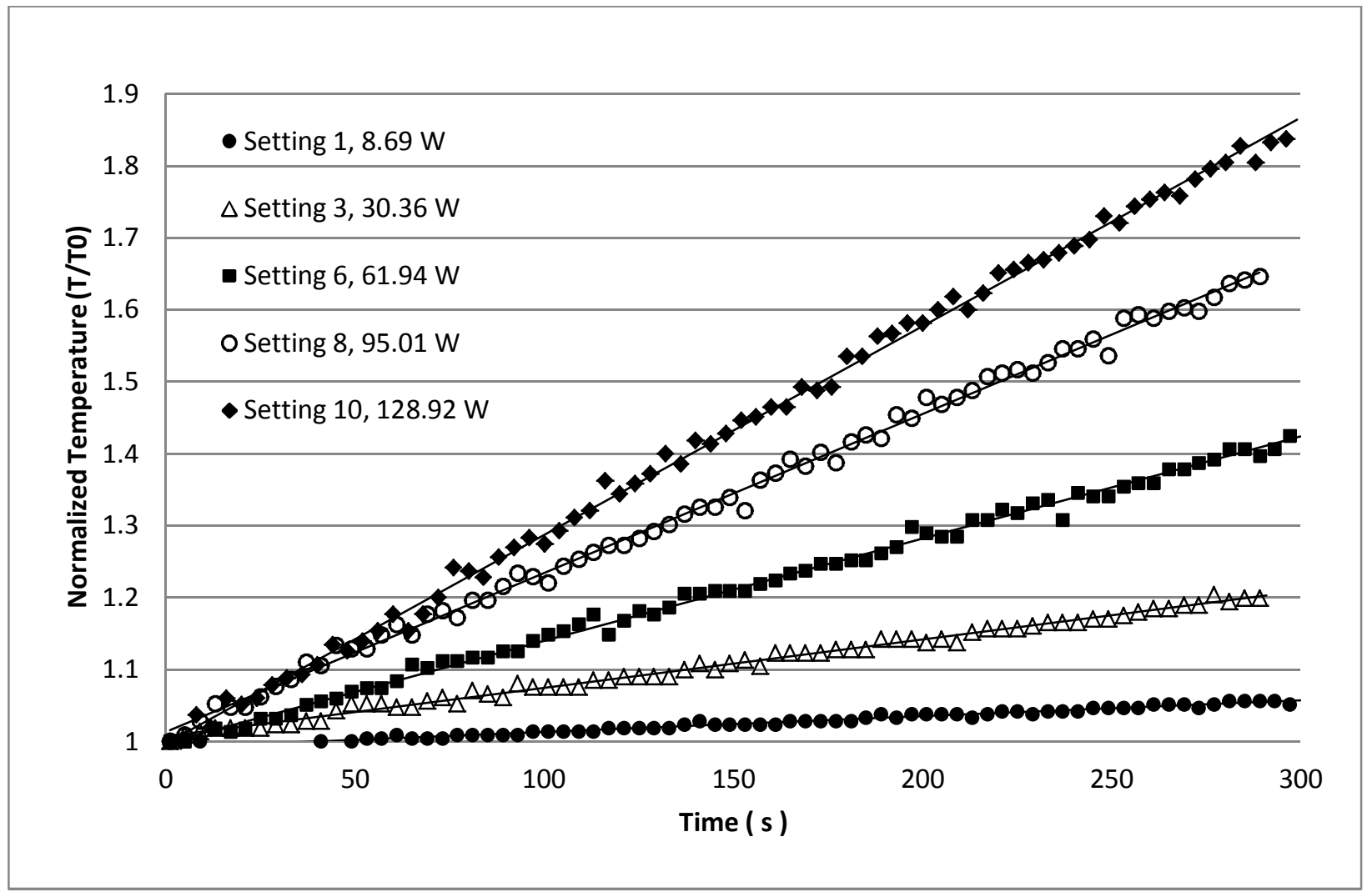

Figure 2. Calorimetric data, linear fits and corresponding calculated delivered power for different operational settings, obtained using a Branson 450 analog disruptor. 


\section{Determination of optimal process parameters}

As previously discussed, the treatment conditions required to achieve complete ENM dispersion are highly system specific. The guidelines offered in this protocol are intended as tools for the interpretation of the observed behavior at a given set of sonication conditions and to determine the appropriate parameter modifications to be made in response. The ultimate goal of the optimization procedure is to achieve the desired degree of dispersion with the least possible energy input, in order to minimize unwanted side effects.

The process to determine such conditions is based on a trial-and-error approach. The optimization process must scan parameter values covering a range both above and below a starting point.

The first trial set of sonication conditions for an untreated powder in suspension should be intended to ensure that dispersion can, in fact, be achieved. The starting point of the optimization must therefore provide a high energy input to the suspension. The following table offers a general guideline of starting points for power, time and volume selection (12).

Table 1. Guidelines for optimization starting points.

\begin{tabular}{|c|c|c|c|}
\hline $\begin{array}{c}\text { Energy } \\
\text { Density } \\
(\mathbf{W} \cdot \mathbf{s} / \mathbf{m L})\end{array}$ & $\begin{array}{c}\text { Sample } \\
\text { Volume (mL) }\end{array}$ & Power (W) & Time (s) \\
\hline Below 100 & 10 & 50 & Below 20 \\
\hline 100 to 500 & 10 & 50 & 20 to 100 \\
\hline Over 500 & 10 & 50 & Over 100 \\
\hline
\end{tabular}

The choice for the starting powder concentration is subject to the desired application. It is also possible to start with concentrations higher than the target one and then, if needed, dilute the processed suspension to the desired level. Concentrations in the (50 to 1000) $\mu \mathrm{g} / \mathrm{mL}$ range are commonly used for toxicological studies.

The effectiveness of the applied sonication conditions can be evaluated by measuring the particle size distribution (PSD) of the sonicated suspension. In principle, reduction of the average particle size and polydispersity are indicative of more effective dispersion.

Once a starting set of values for time, concentration and volume has been selected, and keeping all other processing parameters constant, sonication power is varied. As a general guideline:

1) Scan the effect of power in increments of $10 \mathrm{~W}$ both above and below the starting sonication power.

2) Using the power value that yields the smallest mean particle size and lowest polydispersity, proceed to scan for sonication times in increments of $30 \mathrm{~s}$ above and below 
the starting point. Operation in pulsed mode is recommended for sonication times above 1 min, particularly when using small volumes (below $50 \mathrm{~mL}$ ).

3) For the optimized time, power and selected volume, the effect of particle concentration both above and below the concentration stating point, and down to the minimum acceptable concentration for the desired application, may be evaluated if desired. If the optimal concentration is above the desired value for the particular application, the suspension's PSD must be measured after dilution to check for changes.

\section{Operation and maintenance considerations}

\section{Aerosoling and foaming}

If the sonicator probe is not sufficiently immersed in the suspension, it can give rise to surface agitation resulting in nebulization (formation and release of aerosols). This could pose a risk if ENMs or other potentially harmful medium components are released in this manner.

Aerosoling may be indicated by changes in the audible sound pitch during operation, fluctuating power readings or by the appearance of a fine aerosol in the vicinity of the probe. If aerosoling occurs, one should cease operation and lower the probe deeper into the suspension before resuming operation.

Additionally, if surfactants are present, the suspension could foam during sonication and the presence of stable foam in contact with the probe surface will interfere with the delivery of ultrasonic energy to the suspension in a self-limiting process. Pulse mode operation with long off periods will help avoid foaming in samples subject to this effect. Sonication should be ceased in the event that foaming occurs and the foam removed or allowed to dissipate before continuing.

\section{Tip maintenance}

Tip erosion is an unavoidable side effect in direct sonication. When the tip erodes, microscopic tip residues (typically titanium metal) are released from the tip into the sonicated suspension, introducing impurities and potentially contaminating the suspension. Tip erosion also results in a reduced energy output.

If used on a daily basis, tips should be inspected at least once weekly. Tip erosion can be recognized by the appearance of a grayish matting, as opposed to the lustrous appearance of a non-eroded tip (Figure 3).

The adverse effects of erosion can be mitigated by maintaining a well-dressed tip surface. A matted tip can be reconstituted (dressed) by buffing with a fine grit crocus cloth, carbide paper or emery cloth (note: avoid use of conventional "sand paper"). In the case of a removable tip, set the tip perpendicular to the buffing material placed on a hard, flat work surface and work the tip lightly across the grit in a circular pattern until the matte grey finish is replaced by a finely crisscrossed pattern with a metallic appearance. Substantially eroded tips (i.e., those with visible pitting) should be replaced. Refer to sonicator manufacturer recommendations for further tip cleaning details. 
If the probe's tip is removable, frequent operation may eventually loosen the tip resulting in a lower acoustic power conversion and energy yield. The probe tip should be periodically inspected and retightened if necessary.
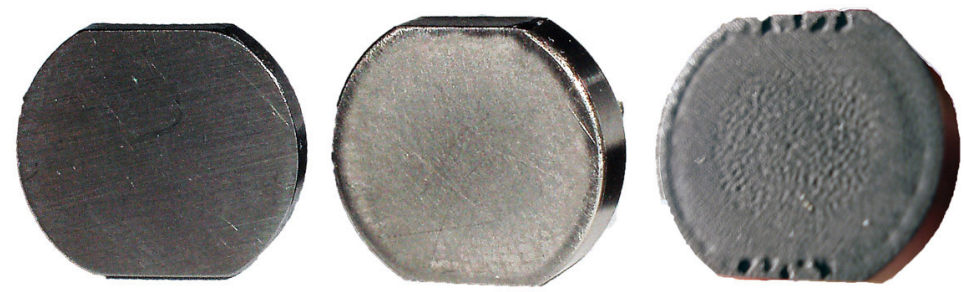

Figure 3. Surface appearance for removable $1 / 2$ " flat tip, ranging from pristine (left), to matted (center), to badly eroded with pitting (right).

\section{Abbreviations}

ENM engineered nanomaterial

PSD particle size distribution

\section{References}

1. Roebben, G., Ramirez-Garcia, S., Hackley, V.A., Roesslein, M., Klaessig, F., Kestens, V., Lynch, I., Garner, C.M., Rawle, A., Elder, A., Colvin, V., Kreyling, W., Krug, H.F., Lewicka, Z., McNeil, S., Nel, A., Patri, A., Wick, P., Wiesner, M.R., Xia, T., Oberdörster, G., and Dawson, K., Interlaboratory reproducibility of size and surface charge measurements on nanoparticles prior to biological impact assessment. $J$. Nanopart. Res., 2011. 13(7): p. 2675-2687.

2. Taurozzi, J.S., Hackley, V.A., and Wiesner, M.R., Ultrasonic dispersion of nanoparticles for environmental, health and safety assessment - Issues and recommendations. Nanotoxicology, 2011. 5(4): p. 711-729.

3. Taurozzi, J.S., Hackley, V.A., and Wiesner, M.R., Reporting guidelines for the preparation of nanoparticle dispersions from dry materials. Special Publication 1200-1, National Institute of Standards and Technology, Gaithersburg, MD, June 2012.

4. Berlan, J. and Mason, T.J., Sonochemistry: from research laboratories to industrial plants. Ultrasonics, 1992. 30(4): p. 203-212.

5. Brown, B. and Goodman, J., High Instensity Ultrasonics: Industrial Applications. 1965: D. Van Nostrand Company.

6. Puskar, A., The use of High Intensity Ultrasonics. 1982: Elsevier Scientific Publishing Company.

7. Lorimer, J.P., Mason, T.J., Cuthbert, T.C., and Brookfield, E.A., Effect of ultrasound on the degradation of aqueous native dextran. Ultrason. Sonochem., 1995. 2(1): p. S55-S57.

8. Wang, J., Wu, J., Zhang, Z.H., Zhang, X.D., Wang, L., Xu, L., Guo, B.D., Li, H., and Tong, J., Sonocatalytic damage of Bovine Serum Albumin (BSA) in the presence of 
nanomater Titanium Dioxide (TiO2) Catalyst. Chin. Chem. Lett., 2005. 16(8): p. 11051107.

9. Suslick, K.S., Ultrasound: Its Chemical, Physical and Biological Effects. 1988: VCH Publishers.

10. Mason, T.J., Sonochemistry : Theory, applications and uses of ultrasound in chemistry. 1989: Ellis Horwood.

11. Mason, T.J. and Peters, D., Practical sonochemistry: Power ultrasound uses and applications. 2003.

12. Hielscher, T., Ultrasonic production of nano-sized dispersions and emulsions (http://arxiv.org/ftp/arxiv/papers/o708/0708.1831.pdf), in Dans European Nano Systems Workshop. 2005: Paris, France. 